\title{
Do Secular Trends in Skeletal Maturity Occur Equally in Both Sexes?
}

\author{
Dana L. Duren PhD, Ramzi W. Nahhas PhD, \\ Richard J. Sherwood PhD
}

Published online: 26 February 2015

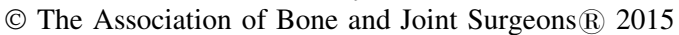

\begin{abstract}
Background Skeletal maturity assessment provides information on a child's physical development and expectations based on chronological age. Given recently recognized trends for earlier maturity in a variety of systems, most notably puberty, examination of sexspecific secular trends in skeletal maturation is important. For the orthopaedist, recent trends and changes in developmental timing can affect clinical management
\end{abstract}

Funding for this study was obtained from the National Institutes of Health: the National Institute for Arthritis and Musculoskeletal and Skin Diseases (R01AR055927) (DLD/WCC) and the Eunice Kennedy Shriver National Institute for Child Health and Human Development (R01HD056247) (DLD) and R01HD012252 (SAC).

All ICMJE Conflict of Interest Forms for authors and Clinical Orthopaedics and Related Research ${ }^{\mathbb{R}}$ editors and board members are on file with the publication and can be viewed on request.

Each author certifies that his or her institution approved the human protocol for this investigation, that all investigations were conducted in conformity with ethical principles of research, and that informed consent for participation in the study was obtained.

This work was performed at Wright State University Boonshoft School of Medicine, Dayton, OH, USA.

Electronic supplementary material The online version of this article (doi:10.1007/s11999-015-4213-1) contains supplementary material, which is available to authorized users.

D. L. Duren $(\bowtie)$, R. W. Nahhas, R. J. Sherwood

Division of Morphological Sciences and Biostatistics,

Department of Community Health, Boonshoft School of

Medicine, Wright State University, 3171 Research Boulevard,

Kettering, Dayton, OH 45420, USA

e-mail: dana.duren@wright.edu

D. L. Duren

Department of Orthopaedic Surgery, Sports Medicine and Rehabilitation, Boonshoft School of Medicine, Wright State

University, Dayton, OH, USA (eg, treatment timing) if they are currently based on outdated sources.

Questions/purposes (1) Has the male or female pediatric skeleton experienced a secular trend for earlier maturation over the past 80 years? (2) Do all indicators of maturity trend in the same direction (earlier versus later)?

Methods In this retrospective study, a total of 1240 children were examined longitudinally through hand-wrist radiographs for skeletal maturity based on the Fels method. All subjects participate in the Fels Longitudinal Study based in Ohio and were born between 1930 and 1964 for the "early" cohort and between 1965 and 2001 for the "recent" cohort. Sex-specific secular trends were estimated for (1) mean relative skeletal maturity through linear mixed models; and (2) median age of maturation for individual maturity indicators through logistic regression and generalized estimating equations.

Results Overall relative skeletal maturity was significantly advanced in the recent cohort (maximum difference of 5 months at age 13 years for girls, 4 months at age 15 years for boys). For individual maturity indicators, the direction and magnitude of secular trends varied by indicator type and sex. The following statistically significant secular trends were found: (1) earlier maturation of indicators of fusion in both sexes (4 months for girls,

\section{R. W. Nahhas}

Department of Psychiatry, Boonshoft School of Medicine,

Wright State University, Dayton, OH, USA

\section{R. J. Sherwood}

Department of Pediatrics, Boonshoft School of Medicine, Wright State University, Dayton, OH, USA 
3 months for boys); (2) later maturation of indicators of projection in long bones in both sexes ( 3 months for girls, 2 months for boys); (3) earlier maturation of indicators of density (4 months) and projection ( 3 months) in carpals and density in long bones (6 months), for girls only; and (4) later maturation of indicators of long bone shape (3 months) for boys only.

Conclusions A secular trend has occurred in the tempo of maturation of individual components of the pediatric skeleton, and it has occurred in a sex-specific manner. The mosaic nature of this trend, with both earlier and later maturation of individual components of the skeletal age phenotype, calls for greater attention to specific aspects of maturation in addition to the overall skeletal age estimate. The Fels method is currently the most robust method for capturing these components, and future work by our group will deliver an updated, user-friendly version of the Fels assessment tool. Clinical Relevance Appreciation of sex-specific secular changes in maturation is important for clinical management, including treatment timing, of orthopaedic patients, because children today exhibit a different pattern of maturation than children on whom original maturity assessments were based (including Fels and Greulich-Pyle).

\section{Introduction}

Skeletal age is an important biological marker of maturity in children, providing a window into bone metabolism and health not captured by bone mass or size. Timing of skeletal maturation varies among children and, for the most part, this variation is normal. However, population-level secular trends toward earlier maturity have been documented in a number of maturation markers, including those for the skeleton [7, 9]. A trend for earlier maturity of the skeleton has implications for overall bone health during childhood and possibly tracking into adulthood. Precocious skeletal maturation, as observed in conditions such as precocious puberty and obesity, can lead to short stature, whereas delayed maturation can have the opposite effect $[5,14]$. To the orthopaedist, skeletal maturity gives a sense of how much time remains for growth in the patient and aids in decision-making of treatment timing.

A secular trend toward accelerated skeletal maturation has been observed in boys and girls in the United States [3], Australia [17, 21], and Portugal [6]. Speculations of the origins of such a trend include rapidly changing environmental factors such as chemical exposure [2, 4, 26] and dietary shifts and a concomitant trend for increased adiposity in children [13].

The "skeletal age" phenotype provides an important snapshot of the developing skeleton. It can signal a delay or acceleration of maturation informing the clinician of underlying hormonal or other constitutional issues. The developmental processes of bone encompassed within the skeletal maturity phenotype (such as that captured by the Fels method [23]) are complex and include initiation of ossification, shape changes within elements, the appearance of radiopaque densities, formation of bony projections, and epiphyseal fusion. Although aspects of overall skeletal maturation will be heavily influenced by a common developmental environment, individual indicators will also respond to signals unique to that element. There is no a priori reason to believe that changes in the tempo of maturation for one component will be equivalent across all components. It is currently unknown whether individual aspects of bone development have experienced the trend toward earlier maturation seen in the global skeletal age phenotype. The current study examines secular trends and sexual dimorphism in overall skeletal age and in the ages at which children advance through stages of development in the bones of the hand and wrist.

We answered the following questions: (1) Has the male or female pediatric skeleton experienced a secular trend for earlier maturation over the past 80 years? (2) Do all indicators of maturity trend in the same direction (earlier versus later)?

\section{Patients and Methods}

\section{Study Sample}

Subjects in the current study are participants of the Fels Longitudinal Study, the world's largest and longest running study of human growth, development, and body composition change over the lifespan [22]. Initiated in 1929, the Fels Longitudinal Study followed, and continues to follow, participants from birth through the entirety of their lives. Subjects are examined at our research facility at regular intervals. Subjects are primarily of European ancestry and reside in southwest Ohio or surrounding regions. Subjects in this sample were not selected for any disease or bonerelated trait and are considered normal and healthy.

Participants in the current investigation include 1240 children born between 1929 and 2001 who were examined serially between birth and 19 years of age. The sample was divided into two cohorts based on the approximate end of the "baby boom era" (approximately 1965). In the "early cohort" (born between 1929 and 1964), there are 342 boys and 333 girls. In the "recent cohort" (born between 1965 and 2001), there are 293 boys and 272 girls (Table 1). Of the 1240 children, a total of 19,283 radiographs of the left handwrist were obtained with participant and parental consent. Individual participants had between one and 37 radiographs between birth and 19 years of age with an average of 16 radiographs per person. After 1987, no radiographs were 
Table 1. Distribution of children in the sample

\begin{tabular}{|c|c|c|c|c|c|c|c|c|}
\hline \multirow[t]{2}{*}{ Sex } & \multicolumn{4}{|c|}{ Earlier cohort } & \multicolumn{4}{|l|}{ Recent cohort } \\
\hline & Birth year & Number & Radiographs & Age & Birth year & Number & Radiographs & Age \\
\hline \multirow[t]{2}{*}{ Girls } & 1929-1964 & 333 & 6626 & Birth to 19 years & 1965-2001 & 272 & 2566 & Birth to 19 years \\
\hline & & & & & 1965-1987* & 175 & 2201 & \\
\hline \multirow[t]{2}{*}{ Boys } & 1929-1964 & 342 & 7169 & Birth to 19 years & $1965-2001$ & 293 & 2922 & 1 month to 19 years \\
\hline & & & & & 1965-1987* & 189 & 2493 & \\
\hline
\end{tabular}

* After 1988, children were not radiographed before age 8 years. Therefore, for traits measured before target age 8 years, only children born before 1988 were included.

obtained for children younger than age 8 years. All procedures used in this study have been approved by the Wright State University institutional review board.

\section{Skeletal Maturity Assessment}

Skeletal maturity was assessed using the Fels method, a maximum likelihood-based method that provides both a skeletal age estimate and a SE [18, 23]. The Fels method is based on objective assessment of as many as 98 individual indicators of bone maturity from 29 bones of the handwrist depending on the child's age. Of these, 85 are categorical and 13 are continuous (epiphyseal/metaphyseal ratios). The Fels method is distinct from, and in our opinion advantageous over, the somewhat subjective Greulich-Pyle atlas method in that the clinician can get a sense of how (for what reasons) a child is accelerated or delayed in their maturation.

Indicators of skeletal maturation in the Fels method can be divided into biologically meaningful groups. We grouped the 85 categorical indicators into five sets: ossification, radiopaque densities, bony projection, shape changes, and fusion (Table 2).

In this article, we use the term "skeletal age" to refer to an overall measure of skeletal maturity and "maturity" for an individual indicator to mean the completion of maturation of a given bone trait.

\section{Statistical Analysis}

To examine the secular trend in our sample for the overall skeletal age phenotype, we estimated mean relative skeletal age (skeletal age - chronological age) as a function of chronological age using a natural spline with 5 degrees of freedom, by sex and cohort, through a linear mixed model [15].

We used repeated-measures logistic regression and generalized estimating equations $[16,19]$ to estimate, for each trait, the median and interquartile range (IQR) of the age of maturation (expressed in months) and to test whether individual maturity indicators exhibited a secular trends in these quantities. Statistical analysis was performed using the geepack package in R Version 2.13.1 [10, 16, 20, 25].

Most categorical indicators (58 of 85) are binary (immature versus mature), whereas remaining indicators (27) are ordinal (with more than two maturity grades). For some ordinal indicators, transitions between different grades fall into different categories (eg, for U1, moving from grade 1 to 2 represents ossification, whereas maturing to grade 3 represents a shape change). Therefore, for this analysis, we split each ordinal indicator into a set of binary indicators numbering one fewer than the number of grades (n) for that ordinal indicator. For example, for an indicator $\mathrm{X}$ with four grades $(1,2,3,4)$, we created three binary indicators, $X_{1}, X_{2}$, and $X_{3}$, where $X_{i}=1$ if $X>i$. This resulted in a total of 121 binary indicators. Note that each indicator has an age range over which it is included in the set of indicators to be assessed for a child. For indicators for which this range starts before age 8 years, children born after 1987 were not included in the analysis.

For each binary indicator of skeletal maturity, by sex, we ascertained the significance (two-sided, $\alpha=0.05$ ) of differences between cohorts in the median and the IQR of age of maturity and computed associated $95 \%$ confidence intervals. SEs for the median and IQR, which are nonlinear functions of the logistic regression parameters, were derived using the delta method. We adjusted for multiple testing using the Holm-Bonferroni adjustment [12]. The statistical model for each indicator results in estimates of secular shifts in timing (median age) and variation (IQR of age) of maturation (see example in Fig. 1). We focus on trends in timing, but also include results for variation (Table 3; Supplemental Table 1 [Supplemental materials are available with the online version of $\left.\mathrm{CORR}^{\circledR}.\right]$ ).

Based on these analyses, we computed the proportion of indicators exhibiting a statistically significant secular trend in maturational timing (earlier or later median age). Additionally, for each indicator group, we used permutation tests to test the null hypotheses of no secular trend in the 
Table 2. Description of five maturation categories for grouping 85 categorical Fels indicators*

\begin{tabular}{lcc}
\hline Maturation category & Brief description & Fels indicators included (and specific grades, if applicable) \\
\hline Ossification & Postnatal ossification of the & AS1, C1, H1, L1, P1, S1, TPD1, TPM1, TRI1, DP31, DP51, \\
& carpals and epiphyses & MET11, MET31, MET51, MP31, MP51, PP11, PP31, PP51, R1, \\
Density (radiopaque) & Appearance of specific radiopaque & H4, TPD2, TPD5, TPD7, TPM3, TPM4, TRI4, MET13, MET33, \\
& densities in a bone & MET53, PP14, PP34, PP54, R3, R4 \\
Projection & Outward bony projections & C4, H3, L1, L2, S3, TPD6, TPM2, TPM2, TPM5, TRI3, \\
& apparent on radiograph & DP33, DP53, MET15, MET16, MET34, MET54, MP33, MP53, \\
Shape & PP17, R5 & C2, C3, C3, H1, H2, L1, L1, S1, S1, S2, S2, S2, TPD3, TPD4, TPM1, \\
& The changes a bone & TPM1, TPM1, TRI, TRI2, TRI2, MET11, MET14, MET31, \\
& & MET31, MET56, MP34, MP54, PP13, PP16, PP33, PP36, PP53, \\
Fusion & R1, U1 & DP14, DP14, DP34, DP34, DP54, DP54, MET17, MET17, MET35, \\
& Stages of epiphyseal fusion & MET35, MET55, MET55, MP35, MP35, MP55, MP55, PP15, \\
& of the long bones in the hand-wrist & PP15, PP35, PP35, PP55, PP55, R6, R6, R6, R7, R7, R7, R8, R8,
\end{tabular}

* Note that some indicators are included in multiple categories, because they span different developmental types at various stages (see Supplemental Table 1 for stage details). See Roche [22] for description of individual indicators.

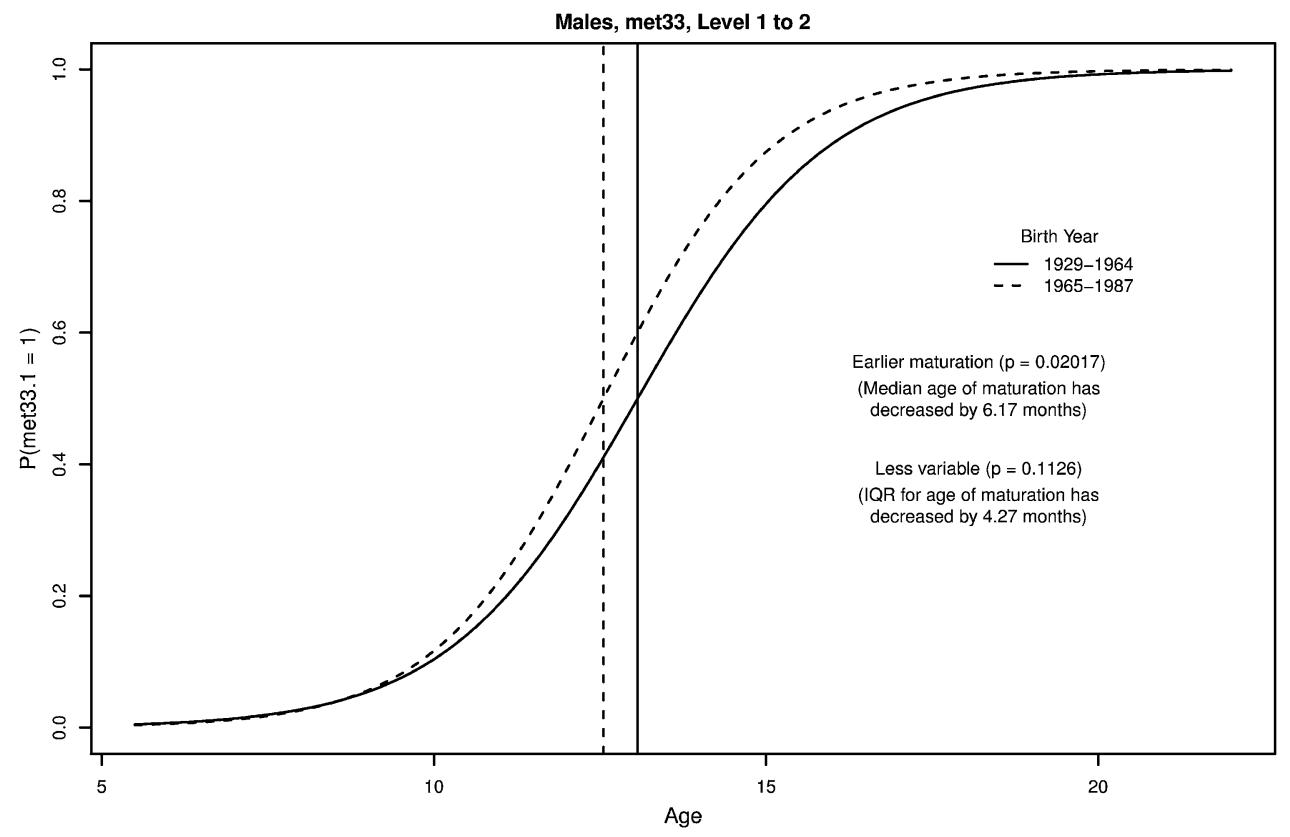

Fig. 1 An example of logistic regression and interpretation of parameters is presented as the probability of maturity of Indicator MET33 (appearance of a radiopaque line or zone within the lateral

margin of the epiphysis of the third metacarpal) in boys in the early (solid line) and recent (dashed line) cohorts.

timing of maturation (ie, no difference between cohorts in the median of the median ages at maturation).

\section{Results}

In answer to our questions: (1) the pediatric skeleton in both boys and girls has experienced a secular trend for earlier skeletal maturity over the past 80 years; and (2) individual indicators of maturity did not all trend in the same direction with some maturing earlier and others later than in previous generations.

\section{Secular Trend in Skeletal Age}

The pattern of relative skeletal age is different for children born before and after 1965 with differences between cohorts 
Table 3. Proportion of binary and ordinal traits that exhibit a significant $(\mathrm{p}<0.05)$ difference in median age at maturation (earlier or later) and in the variability in age at maturation (more or less variable) by sex and trait group*

\begin{tabular}{|c|c|c|c|c|c|c|c|c|c|c|}
\hline \multicolumn{2}{|c|}{ Trait category } & \multirow[t]{2}{*}{ Number } & \multicolumn{4}{|l|}{ Boys } & \multicolumn{4}{|l|}{ Girls } \\
\hline & & & Earlier & Later & More variable & Less variable & Earlier & Later & More variable & Less variable \\
\hline \multirow[t]{2}{*}{ Ossification } & Carpals & 9 & $0 \%$ & $0 \%$ & $11 \%$ & $0 \%$ & $0 \%$ & $0 \%$ & $11 \%$ & $0 \%$ \\
\hline & Long bones & 12 & $0 \%$ & $0 \%$ & $8 \%$ & $0 \%$ & $33 \%$ & $0 \%$ & $0 \%$ & $8 \%$ \\
\hline \multirow[t]{2}{*}{ Density } & Carpals & 7 & $14 \%$ & $29 \%$ & $29 \%$ & $0 \%$ & $57 \%$ & $0 \%$ & $14 \%$ & $0 \%$ \\
\hline & Long bones & 8 & $25 \%$ & $38 \%$ & $0 \%$ & $25 \%$ & $63 \%$ & $25 \%$ & $0 \%$ & $13 \%$ \\
\hline \multirow[t]{2}{*}{ Projection } & Carpals & 10 & $0 \%$ & $20 \%$ & $20 \%$ & $10 \%$ & $30 \%$ & $20 \%$ & $10 \%$ & $0 \%$ \\
\hline & Long bones & 10 & $30 \%$ & $30 \%$ & $20 \%$ & $20 \%$ & $20 \%$ & $20 \%$ & $0 \%$ & $10 \%$ \\
\hline \multirow[t]{2}{*}{ Shape } & Carpals & 20 & $5 \%$ & $15 \%$ & $10 \%$ & $5 \%$ & $5 \%$ & $10 \%$ & $20 \%$ & $5 \%$ \\
\hline & Long bones & 14 & $0 \%$ & $36 \%$ & $21 \%$ & $0 \%$ & $14 \%$ & $7 \%$ & $7 \%$ & $0 \%$ \\
\hline Fusion & Long bones & 31 & $48 \%$ & $0 \%$ & $7 \%$ & $7 \%$ & $68 \%$ & $0 \%$ & $23 \%$ & $0 \%$ \\
\hline
\end{tabular}

* Percentages are rounded to the nearest whole number.

as great as 5 months (Fig. 2). There was a significant cohort $x$ age interaction for both sexes $(p<0.01)$, indicating the magnitude of the secular change varied across chronological ages. The recent cohort had skeletal age approximately equal to, or more advanced than, the earlier cohort at all ages with the largest difference during puberty. Recent boys showed earlier maturation between ages 0 to 8 years and 10 to 18 years (Fig. 2A) with the maximum difference of 4 months advanced skeletal age occurring at age 15 years. Recent girls exhibited earlier maturation between the ages of 4 and 17 years (Fig. 2B) with the maximum difference of 5 months advanced skeletal age occurring at age 13 years.

\section{Secular Trend in Individual Indicators}

Although there is an overall secular trend toward earlier skeletal development, the direction and magnitude of secular change vary between individual maturity indicator types and between indicators of the same type.

The proportions of indicators with a significant $(\mathrm{p} \leq 0.05)$ secular trend in median or IQR of age of maturation ranged from $0 \%$ (no secular trend) to between $5 \%$ of carpal shape indicators and $68 \%$ of fusion indicators maturing earlier and between $7 \%$ of long bone shape indicators and $36 \%$ of long bone density indicators maturing later (Table 3). The magnitude of the trend in median age of maturation differed by indicator group (Fig. 3) (for details on secular trends for each indicator, see Supplemental Table 1).

\section{Boys}

For boys, within indicator categories the percentage of indicators exhibiting earlier maturation (by 3 months) in the recent cohort was as high as $48 \%$ (fusion indicators), and the percentage of indicators exhibiting later maturation in the recent cohort was as high as $38 \%$ (density indicators in long bones). There was also substantial variation in the magnitudes of trends in indicator groupings (Fig. 3A). In some cases, there are significant trends in timing of maturation for individual indicators that are opposite relative to the majority in their group. For example, three long bone projection indicators exhibited later maturation (DP33: 5 months, $\mathrm{p}<0.01$; DP53: 7 months, $\mathrm{p}<0.01$; MP33: 6 months, $\mathrm{p}<0.01$ ), and two indicators had significantly earlier maturation (MET16: -10 months, $\mathrm{p}<0.01$; PP17: -8 months, $\mathrm{p}<0.01$ ) (Supplemental Table 1).

Even for indicator groups not demonstrating statistically significant $(\mathrm{p}<0.05)$ secular trends in median age of maturation, some individual indicators have undergone statistically significant trends (Fig. 3A; Supplemental Table 1). For example, although the median change in timing for projections in carpals is close to zero, both TPM2 and TRI3 have undergone statistically significant secular trends toward later maturation exceeding 1 year (14 and 16 months, respectively; $\mathrm{p}<0.01$ for each).

\section{Girls}

For girls, within indicator categories the percentage of indicators exhibiting earlier maturation (by 4 months) in the recent cohort was as high as $68 \%$ (fusion indicators), and the percentage exhibiting later maturation (by 6 months) in the recent cohort was as high as 25\% (density indicators in long bones). Variation in magnitudes of trends within indicator groupings in girls was substantial. In some cases trends in the timing of maturation for individual indicators occur in the opposite direction to the majority in their group. For example, carpal density indicator TPM4 


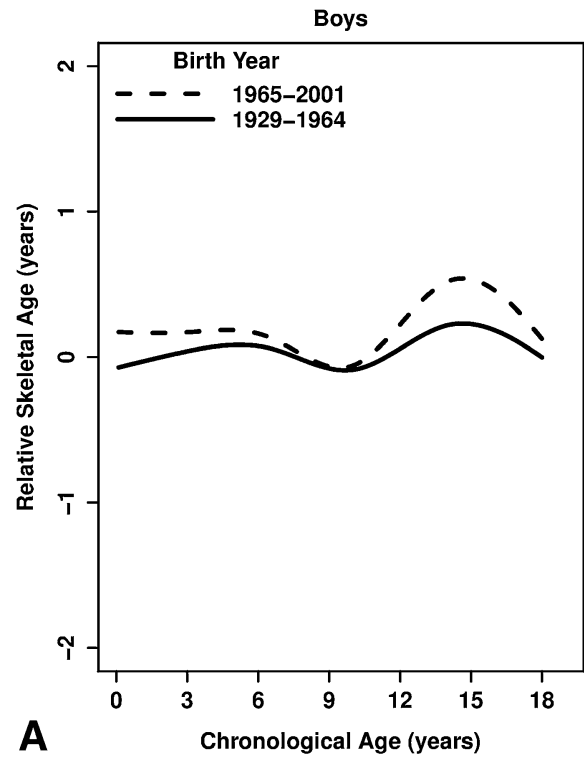

Fig. 2A-B Relative skeletal age (skeletal age - chronological age) versus chronological age is presented by birth cohort in (A) boys and (B) girls. Greater relative skeletal age indicates earlier maturation. The lines estimate the average relative skeletal age at a given chronological age for boys and girls. The early cohort (solid line) has

exhibited slightly later maturation (0.8 months), whereas four indicators had significantly earlier maturation (TPD2: -7 months, $\mathrm{p}<0.01$; TPD5: -4 months, $\mathrm{p}=0.03$; TPM3: -7 months, $\mathrm{p}<0.01$; and TRI4: -8 months, $\mathrm{p}<0.01$ ) (Fig. 3B; Supplemental Table 1).

Indicator groups not demonstrating a statistically significant secular trend in median age of maturation did contain some individual indicators exhibiting significant trends for earlier or later maturation. For example, although the median change in timing for shape in carpals approaches zero, TRI2 has undergone a significant secular trend toward later maturation approaching 2 years $(21$ months, $\mathrm{p}<0.01)$.

\section{Discussion}

The current study examined sex-specific differences in the tempo of maturation of bones in the hand-wrist between two cohorts of children to determine if any or all processes encompassed within the skeletal age phenotype exhibit secular trends. Previous work by other researchers has shown evidence for a secular trend for earlier overall skeletal maturation in recent cohorts $[3,17,21]$. Our data support these studies because we too observe a secular trend toward earlier skeletal maturity in the Fels Longitudinal Study cohort. To our knowledge, ours is the first study to examine specific aspects of maturation of the skeleton independently and present sex-specific trends in maturation.

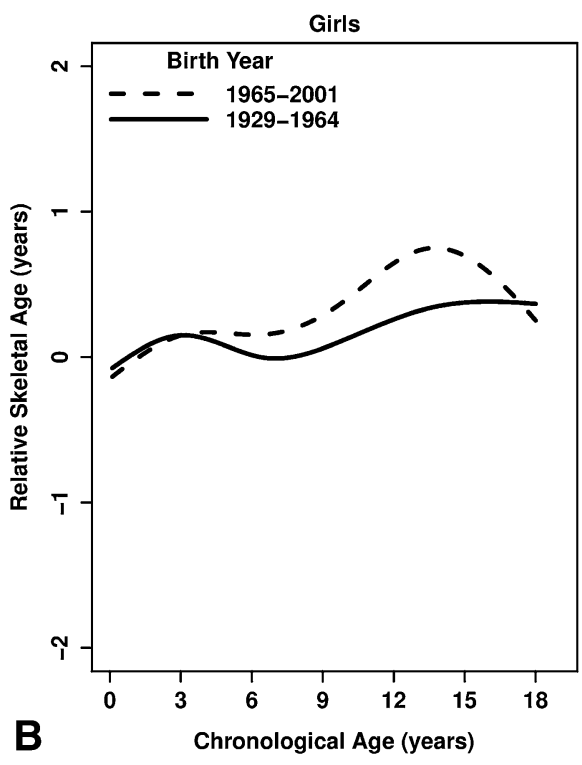

relative skeletal age near zero at all ages (note that this cohort represents the individuals used to develop the Fels method). The recent cohort (dashed line) has relative skeletal age greater than zero, indicating earlier skeletal maturation.

Limitations of the current study include the racial composition of the study, the segregation of traits and categories, and the choice for a cutoff date separating cohorts. First, the study population used for the current investigation is primarily of European descent. It is thus difficult to draw conclusions regarding other races and their sex differences and secular trends. Comparison of our results with secular trends in other races would necessitate radiographic data over many years before and after 1965 in other racial groups. Second, we chose morphological categories for grouping skeletal maturity indicators. The number and type of these maturation categories could be expanded (or collapsed) and were chosen in this analysis for their discrete nature. Likewise, placement of specific indicators into these categories may be debated, but the current construct is appropriate for the questions under investigation. Third, the choice of 1965 (the approximate end of the "baby boom era") as a cutoff date separating the two cohorts was somewhat arbitrary, but represented a tradeoff between two considerations. An earlier cutoff year would have led to serious bias in our conclusions because the original Fels method was calibrated to the data from the individuals born earlier. A later cutoff year would have diminished sample size in the "recent" cohort, particularly in the younger ages.

Sex differences were detected in the secular trend in overall skeletal maturity with recent boys exhibiting more advanced maturation by as much as 4 months at age 15 years and recent girls exhibiting more advanced 

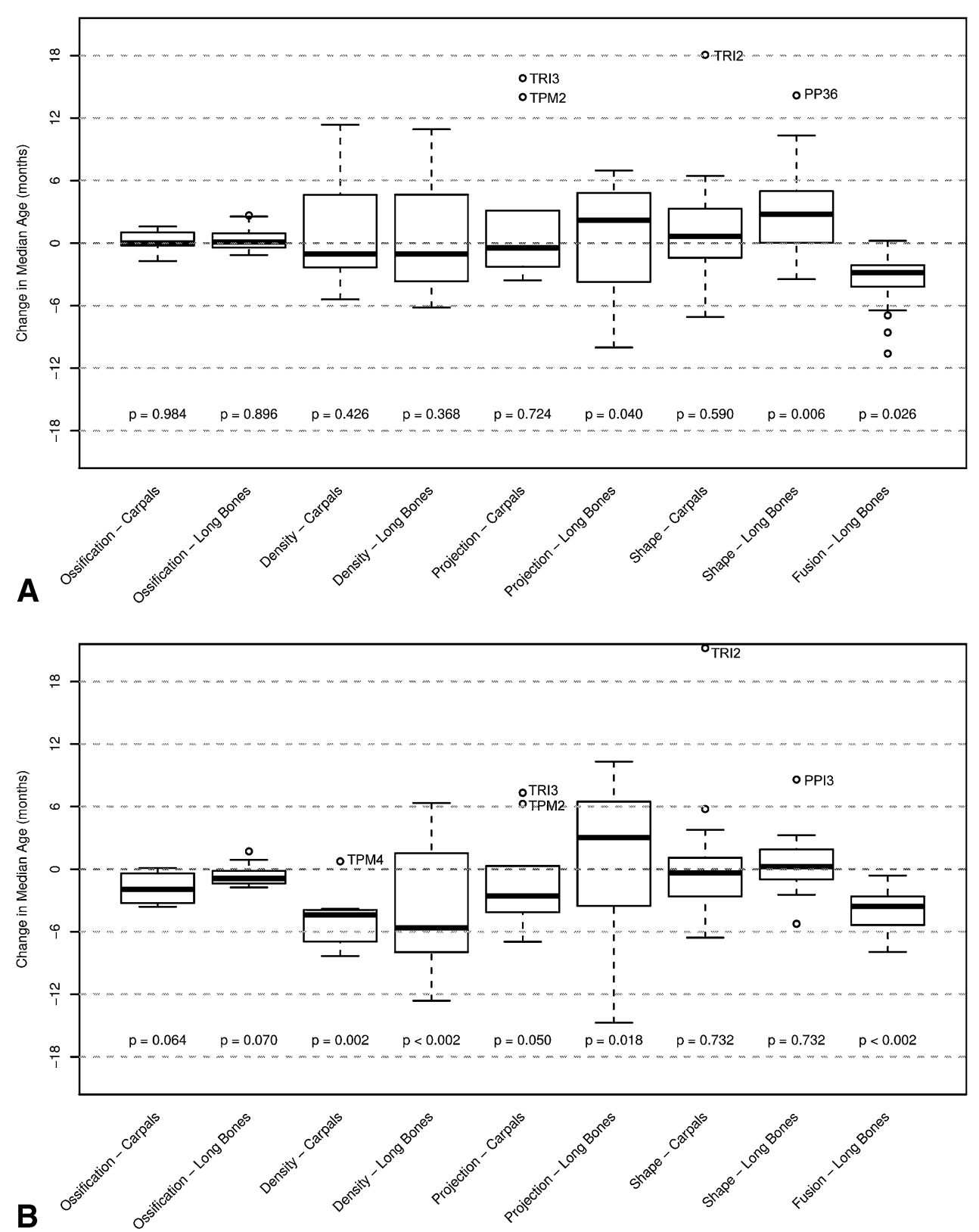

Fig. 3A-B The magnitude of secular trends in each individual indicator group is presented for median age in (A) boys and (B) girls. In each box plot, the thick black line represents the median secular

maturation by as much as 5 months at age 13 years. For both sexes, the largest secular trend was during puberty. The magnitude of the trend in boys is similar to that of Australian boys at age 8 years but very different from the results for girls in the same Australian sample who showed no secular trend in skeletal age [17]. Compared with white adolescents of South Africa, the boys in our sample were similar (South African boys were advanced by 3.4 months), and the girls in our sample demonstrated almost twice the advanced skeletal age of their peers in South Africa (2.0 months advanced) [11]. trend within that indicator group with the corresponding permutation test $\mathrm{p}$ value for testing the null hypothesis that the median is zero.

By subdividing Fels skeletal age into five component parts, we identified a mosaic of trends for earlier and later maturation of the skeleton, depending on the type of indicator examined. Many of these trends differ in direction between boys and girls.

Indicators of maturation in radiopaque densities in our study showed trends for earlier maturation, especially in girls, where the magnitude of change in median age of maturation was close to 6 months earlier in the most recent cohort. Importantly, these radiopaque density indicators are not related to overall bone mineral density (BMD), but 
rather indicate the infilling of the cartilaginous model of a carpal or epiphysis and typically appear at joint surfaces. Although skeletal maturity does not have a one-to-one relationship with bone mass, early (or late) achievement of maturity in specific aspects of the skeleton can influence how and where bone is deposited. It has been shown that timing of puberty has a significant impact on bone strength (BMD/bone mineral content and cross-sectional geometry) in women $[8,24]$.

The most striking secular trend within maturity features is the number of fusion indicators maturing earlier in both boys and girls. Fusion indicators represent changes in stages of epiphyseal closure from initiation to full closure. The primary systemic influence on fusion is sex steroid hormones. The median age of maturation in both boys and girls has trended earlier for the more recent cohort, reflecting secular trends in pubertal timing [1, 13]. In our study, some fusion indicators mature as much as 10 months earlier in the recent cohort. Additionally, although epiphyseal fusion signals the end of longitudinal growth, it is yet unknown whether processes leading to "early" (nonpathological) fusion also accelerate growth before fusion or if bone deposition is altered as well.

\section{Clinical Relevance}

We have demonstrated that consideration of only overall skeletal maturity (as occurs with most skeletal age assessments, including Greulich-Pyle) masks the presence of trends of varying direction and magnitude among individual bone traits. Likewise, estimates of skeletal age may mask differences between trends in the between-individual variability in age of maturation within each subtype of maturity indicator. This becomes relevant in clinical settings where a doctor is measuring the effects of treatment on skeletal maturation without consideration of indicator type. For example, if all other indicators of maturation are on target for chronological age, but early stages of fusion are precocious, the overall skeletal age underestimates the effects of the treatment on skeletal maturity in that child. All methods of bone age assessment are guilty of this masking. The Fels method, however, can be used to identify these component parts, whereas other methods scoring whole bone morphology, and not individual aspects of maturation, cannot.

Given this newly uncovered sex-specific mosaic of secular trends in skeletal maturity, it is imperative that current methods of assessing skeletal maturity, and reference populations on which they are based, be updated. Efforts by our group are underway to do such an update. We are further developing utilities estimating the influence of the separate subtypes of maturation within the Fels assessment for better resolution of what, exactly, is accelerated or delayed in a child with an "abnormal" skeletal age.

Acknowledgments We thank the participants of the Fels Longitudinal Study for their dedication to research. We also thank the faculty and staff who have worked with the Fels Longitudinal Study over its $80+$ years and acknowledge grant support to Stefan A. Czerwinski for a portion of data used in this manuscript, and to W. Cameron Chumlea as Co-PI of the original AR055927. We would like to specifically acknowledge the data collection contributions of Carol Cottom, Sharon Lawrence, and Kimberly Lever.

\section{References}

1. Beunen GP, Rogol AD, Malina RM. Indicators of biological maturation and secular changes in biological maturation. Food Nutr Bull. 2006;27:S244-S256.

2. Bourguignon JP, Franssen D, Gerard A, Janssen S, Pinson A, Naveau E, Parent AS. Early neuroendocrine disruption in hypothalamus and hippocampus: developmental effects including female sexual maturation and implications for endocrine disrupting chemical screening. J Neuroendocrinol. 2013;25:1079-1087.

3. Calfee RP, Sutter M, Steffen JA, Goldfarb CA. Skeletal and chronological ages in American adolescents: current findings in skeletal maturation. J Child Orthop. 2010;4:467-470.

4. Deng F, Tao FB, Liu DY, Xu YY, Hao JH, Sun Y, Su PY. Effects of growth environments and two environmental endocrine disruptors on children with idiopathic precocious puberty. Eur $J$ Endocrinol. 2012;166:803-809.

5. Flor-Cisneros A, Roemmich JN, Rogol AD, Baron J. Bone age and onset of puberty in normal boys. Mol Cell Endocrinol. 2006;254-255:202-206.

6. Freitas D, Malina RM, Maia J, Lefevre J, Stasinopoulos M, Gouveia E, Claessens A, Thomis M, Lausen B. Short-term secular change in height, body mass and Tanner-Whitehouse 3 skeletal maturity of Madeira youth, Portugal. Ann Hum Biol. 2012;39:195-205.

7. Garn SM. The secular trend in size and maturational timing and its implications for nutritional assessment. J Nutr. 1987;117:817-823.

8. Gilsanz V, Chalfant J, Kalkwarf H, Zemel B, Lappe J, Oberfield S, Shepherd J, Wren T, Winer K. Age at onset of puberty predicts bone mass in young adulthood. J Pediatr. 2011;158:100-5, 105.

9. Guo SS, Zeller CM, Chumlea WC, Roche AF, Siervogel RM. Body composition and secular trends in children and young adults: the Fels Longitudinal Study 1929-1996. Am J Clin Nutr. 1997;66:220.

10. Halekoh U, Hojsgaard S, Yan J. The R package geepack for generalized estimating equations. J Statistical Software. 2006;15.

11. Hawley NL, Rousham EK, Norris SA, Pettifor JM, Cameron N. Secular trends in skeletal maturity in South Africa: 1962-2001. Ann Hum Biol. 2009;36:584-594.

12. Holm S. A simple sequentially rejective multiple test procedure. Scandinavian Journal of Statistics. 1979;6:65-70.

13. Kaplowitz PB. Link between body fat and the timing of puberty. Pediatrics. 2008;121(Suppl 3):S208-S217.

14. Kemp SF, Sy JP. Analysis of bone age data from national cooperative growth study substudy VII. Pediatrics. 1999;104:1031-1036.

15. Laird NM, Ware JH. Random-effects models for longitudinal data. Biometrics. 1982;38:963-974.

16. Liang KY, Zeger SL. Longitudinal data analysis using generalized linear models. Biometrika. 1986;73:13-22.

17. Lin NH, Ranjitkar S, Macdonald R, Hughes T, Taylor JA, Townsend GC. New growth references for assessment of stature and skeletal maturation in Australians. Aust Orthod J. 2006;22:1-10. 
18. Nahhas RW, Sherwood RJ, Chumlea WC, Duren DL. An update of the statistical methods underlying the FELS method of skeletal maturity assessment. Ann Hum Biol. 2013;40:505-514.

19. Prentice RL, Zhao LP. Estimating equations for parameters in means and covariances of multivariate discrete and continuous responses. Biometrics. 1991;47:825-839.

20. R Development Core Team. R: A Language and Environment for Statistical Computing. Vienna, Austria: R Foundation for Statistical Computing; 2012.

21. Ranjitkar S, Lin NH, Macdonald R, Taylor JA, Townsend GC. Stature and skeletal maturation of two cohorts of Australian children and young adults over the past two decades. Aust Orthod J. 2006;22:47-58.

22. Roche AF. Growth, Maturation and Body Composition: The Fels Longitudinal Study 1929-1991. Cambridge, UK: Cambridge University Press; 1992.
23. Roche AF, Chumlea WC, Thissen D. Assessing the Skeletal Maturity of the Hand-Wrist: Fels Method. Springfield, IL, USA: Charles C. Thomas; 1988.

24. Seselj M, Nahhas RW, Sherwood RJ, Chumlea WC, Towne B, Duren DL. The influence of age at menarche on cross-sectional geometry of bone in young adulthood. Bone. 2012;51:38-45.

25. Yan J, Fine J. Estimating equations for association structures. Stat Med. 2004;23:859-874.

26. Zawatski W, Lee MM. Male pubertal development: are endocrinedisrupting compounds shifting the norms? $J$ Endocrinol. 2013;218:R1-12. 